\title{
FLT3 Receptor Heterogeneity Strictly Specifies the Dimensions of Malignant Transformation Events in Acute Myeloid Leukemia
}

\author{
*Lawrence M Agius \\ Department Of Pathology, University Of Malta Medical School, Europe
}

Received: October 25, 2017; Published: October 31, 2017

*Corresponding author: Lawrence M Agius, Department Of Pathology, Mater Dei Hospital, Tal-Qroqq, University Of Malta Medical School, Msida, Malta Europe, 27 “Ballarat” Guzeppe Caruana Street, Tal-Virtu, Rabat, Rbt09, Malta, Europe, Tel: 356-21451752; Email: lawrence.agius@um.edu.mt

\begin{abstract}
Qualitative mutational events act synergistically with increases in mutated FLT3 receptors in the plasma-membrane in inducing constitutive activation of the receptors in terms of such indices as auto phosphorylation of the tyrosine kinase domains. Dimerization of the FLT3 sub-units and diminished "repulsive" dysfunctions of the juxtamembrane domain allow for permissive receptor activation. Incremental numbers of mutated receptors may strictly characterize the nature of origin of the malignant transformation event in terms of both synergic and compensatory mechanisms that directly modulate and further impact the hematopoietic progenitor cell subpopulations and also renewal of the hematopoietic stem populations in the bone marrow. Blast cell generation proliferation and impaired differentiation programs come to account for a great degree of heterogeneity in hematopoietic ally-related cell types in response to agents with tyrosine kinase inhibitory action.
\end{abstract}

Abbreviations: AML: Acute Myeloid Leukemia; ITDs: Internal Tandem Duplications; TKIs: Tyrosine kinase inhibitors; WT: Wild Type; GFI: Growth Factor Independence; SKY: Spleen Tyrosine kinase

\section{Introduction}

Stem cell hematopoietic replacement or renewal, and inhibitory effects on proliferation or contrasting maturation of progenitor cells are distinct stages and essentially highly distinctive process mechanisms in leukemogenesis. As such, the development of Internal Tandem Duplications (ITDs) in the FLT3 mutant cell juxtamembrane domain, in a large subset of Acute Myeloid Leukemia (AML) patients, has to be considered within such a differential context that is reflected in complete remission, disease-free survival, relapse rate and overall survival of AML patients with ITDs. FLT3ITDs account for up to $25 \%$ of AML cases; the transcription factor ATF4 in these patients is essential for FLT3-ITD-induced autophagy [1]. The development of FLT3-targeted inhibitors constitutes an important paradigm shift in managing patients with very aggressive FLT3-mutated AML [2]. AML is highly heterogeneous and involves immature myeloid cell proliferation and bone marrow failure [3]. Allogeneic stem-cell transplantation decreases the risk of AML recurrence compared to standard chemotherapy but is associated with the risk of serious complications [4]. AML is the most common acute leukemia in adults, and treatment options, particularly in the elderly, are limited [5].

\section{Autophosphorylation}

Oncogenic forms of FLT3 are important therapeutic targets in AML but clinical responses to small-molecule kinase inhibitors are short-lived due to rapid emergence of resistance arising from point mutations or compensatory increases in FLT3 expression [6]. The complexity of autophosphorylation events in the two intra-cellular tyrosine kinase domains implicates both FLT3-ITD mutant cells and also cells with wild-type FLT3 in the development of constitutive activation of the FLT3 receptors. It is hypothesized that FLT3-ITD leukemia cells exhibit mechanisms of intrinsic signaling adaptation to TKI treatment that are related to an incomplete response [7]. It is further to such considerations that various different adaptor proteins are also involved in the activation of different cellular pathways and activators that lead to impaired maturation and increased proliferation of progenitor cells.

The ITDs are associated with preserved reading frame and involve non-ligand activation of the FLT3 receptors in a contextual referential system that involves often groups of three or more duplicated head-to-tail insertions of tandem duplications with 
the stabilization of phosphorylation or open configuration of the tyrosine kinase domains. The ITDs are located to the juxtamembrane domain of the individual receptor in a manner that also implicates Dimerization of FLT3 receptor homodimers.

\section{Constitutional Activation}

The prognosis in AML patients could be further stratified by different mutation combinations and hence the value of nextgeneration sequencing for genomic classification [8]. Dimensions of constitutive activation of the FLT3-ITDs are also reflected in other tyrosine-kinase receptor type III family in a manner that wellillustrates the high levels of homology between various members of this large receptor family. Such high degrees of mechanistic variability in down-stream pathway effects indicate a range of nonspecific targeting of mediators within the potential scope of biologic and clinical consequences of tyrosine kinase activation modulation.

Systems of therapeutic intervention with tyrosine kinase inhibitors (TKIs) indicate that FLT3-WT (wild-type), as constitutively activated, is highly significant in terms comparable to FLT3-ITDs mutant receptors. As such, concepts of constitutive activation of the FLT3 receptors in general and of FLT3-WT/ITD receptors, in particular, also include excessive numbers of these receptors traversing the plasma-membrane of the cells. Within further realization model systems, the signal transmutations of the FLT3 receptors are profoundly influenced by numerous potential parameters of control and modulation in signal transduction. FLT3ITD AML usually responds poorly to conventional therapies and may become resistant to TKIs due to molecular bypass mechanisms [9].

\section{Tyrosine Kinase Domains}

The tyrosine kinase domains that are autophosphorylated and activated lead to an essential stage model that mechanistically can affect proliferation, maturation and even apoptosis of blast cells in both the bone marrow and peripheral blood of AML patients. Degrees of synergism may also include FLT3-ITD and FLT3-TKD (where mutations are present in the tyrosine kinase domains) and are generally exclusive phenomena and appear not generally applicable to the biology of FLT3-mutant AML cells. TKI treatment increases the surface expression through up-regulation of FLT3 and glycosylation of FLT3-ITD and FLT3-D935 Y mutants [10].

FLT3-ITDs are a negative prognostic factor in AML patients particularly in patients over 60 years of age; such a modulated series of effects is reflected in results of studies examining the potential therapeutic benefits of administered TKIs as single agents or when combined with standard chemotherapy. The use of allogeneic stem cell transplantation has improved outcome in FLT3ITDs patients especially in terms of relapse rates, including also AML patients treated immediately after first relapse. Growth Factor Independence 1 (GFI1) is a negative indicator of AML progression and high levels of GFI1 expression are paralleled by higher FLT3 expression and exhibit a FLT3-ITD signature of gene expression; knock-down of GFI1 expression in vitro leads to decreased FLT3 RNA and down-regulation of FLT3-ITD signaturegenes [11].

\section{Multi-Components}

Considered response of FLT3-mutated hematopoietic cells to small molecule inhibitors is, hence, an overall index of participation of multiple pathways that originate from autophosphorylation of the tyrosine kinase domains and that are reflected in such pathways as inhibited apoptosis and in the activation of intra-nuclear catenin. The multi-component systems of adaptor proteins in particular allow for signal response in the evolutionary course of AML cell proliferation and of impaired maturation beyond the hematopoietic blast stage. Spleen tyrosine kinase (SYK) is activated and increases in FLT3-ITD-positive AML patients and is critical for transformation and maintenance of the leukemia clone in these patients [12].

A critical stage of differentiation appears to be the transition of (CD34+/CD33-) subpopulations of progenitor cells to the (CD34+/CD33+) cell population when considering FLT3 precursor cells in AML patients. The three main groups of mutated FLT3 hematopoietic cells include FLT3-ITDs, and also FLT3-point mutations in the juxtamembrane domain, and the tyrosine kinase domain mutations. FLT3-TKD-mutated AML patients have a more favorable prognosis clinically when compared with patients with FLT3-ITD mutations [13]. As such, an essentially significant degree of heterogeneity is found within the complex phenomenon of FLT3mutated hematopoietic progenitor cell populations considered and is contributory to AML leukemogenesis. The complex heterogeneity of AML has seriously hampered the development of a curative treatment; mono-therapy is associated with resistance due to the parallel signaling circuitry involving also MAPK and mTOR [14]. Whereas FLT3-ITD cells tend to target myeloid-type cells, FLT3TKDs are associated with a preferential selection of lymphoid-type precursor cells.

\section{Autophosphorylation}

The essential absence of stop-codons in both FLT3-ITD and FLT3-TKD cells affecting especially the internal tandem duplications in the former group of mutated receptors is fully consistent with excessive signaling as a hallmark of the constitutive receptor activation and tyrosine kinase autophosphorylation. Dynamics of constitutive phosphorylation of the FLT3-mutated receptors are reflected within contexts of auto-stimulation that primarily include excessive suppression of the "repulsive forces" that affect the juxtamembrane domain of the receptor in patients with FLT3-ITDs. Also, glycosylation of the extra-cellular domain of the FLT3 cells, whether FLT3-ITD or WT, may possibly be implicated not only in the trans-membrane insertion of the FLT3 receptors but also as implemented component in response to extra-cellular stimuli that may synergistically cooperate with constitutive receptor activation and phosphorylation of the tyrosine kinase domains.

Andrographolide (an active component of Andrographis paniculata) suppresses MV4-11 cell proliferation through inhibition of FLT3 signaling with also inhibition of fatty acid synthesis and cellular iron uptake [15]. FLT3 LIGAND The FLT3 receptor ligand FL functionality is mechanistically homologous to the KIT receptor and dynamics of each ligand type and is projected towards the 
further creation of established states of autophosphorylation of the tyrosine kinase domain. In this sense, TKIs may be expected to impact the constitutive activation of the blast cells in terms of ongoing dimensions of proliferation and impaired maturation of the hematopoietic progenitor cells in patients with AML.

Results of the non-responsive sub-populations of hematopoietic progenitor cells may in part arise from the marked heterogeneity of pathway response and inclusion as terms of effect arising from constitutive activation and autophosphorylation of the FLT3 receptors. High expression of proviral integration site for Moloney murine leukemia virus-1 (PIM-1), a serine/threonine kinase, is a high risk independent prognostic factor in AML [16]. The SOX4 transcription factor is not only an independent prognostic factor in AML but also an important molecular oncogenic agent in leukemogenesis [17].

High expression of Inhibin-Beta A (a ligand of the transforming growth factor Beta super family) is an adverse prognostic marker for de novo AML [18]. As such, incremental numbers of FLT3 receptors that traverse the cell membrane may potentially prove a dynamic interplay with tyrosine kinase activation in further oncogenesis and synergistically potentiate ongoing progression of the AML cell subpopulations. Indeed, further synergistic compensatory increases in FLT3-ITDs may prove instrumental in augmenting dimensions of malignant transformation events that include stem cell subpopulations generating mutated progenitor hematopoietic cells.

\section{Concluding Remarks}

In considering the significant degrees of heterogeneous subpopulations of FLT3 receptors one may expect that synergistic cooperative effects arising from many components downstream implicate effective reconstruction of compensatory systems of response; the compensatory effects include also an increase in mutated FLT3 receptors in the plasma membrane in a manner that itself specifically promotes autophosphorylation of the tyrosine kinase domains.

\section{References}

1. Heydt Q, Larrue C, Saland E, Bertoli S, Sarry JE, et al. (2017) Oncogenic FLT3-ITD supports autophagy via ATF4 in acute myeloid leukemia. Oncogene.

2. Gallogly MM, Lazarus HM, Cooper BW (2017) Midostaurin: a novel leukemia and systemic mastocytosis. Ther Adv Hematol 8(9): 245-261.

3. Rostami S, Nadali F, Alibakhshi R, Zaker F, Nasiri N, et al. (2017) Aberrant methylation of APAF-1 gene in acute myeloid leukemia patients. Int J Hematol Oncol Stem Cell Res 11(3): 225-230.
4. Miyamoto T, Kikushige Y, Yoshimoto AG (2017) Frontline treatment of AML in adults. Rinsho Ketsueki 58(10): 1884-1894.

5. Stanssfield LC, Pollyea DA (2017) Midostaurin: a new oral agent targeting FLT3-mutant acute myeloid leukemia. Pharmacotherapy.

6. Weisberg EL, Schauer NJ, Yang J, Lamberto I, Doherty L, et al. (2017) Inhibition of USP10 induces degradation of Oncogenic FLT3. Nat Chem Biol.

7. Bruner JK, Ma HS, Li L, Qin ACR, Rudek MA, et al. (2017) Adaptation to TKI treatment reactivates ERK signaling in tyrosine kinase-driven leukemias and other malignancies. Cancer Res 77(20): 5554-5563.

8. De Noronha TR, Mitne-Neto M, Chauffaille ML (2017) Mutational profiling of acute myeloid leukemia with normal cytogenetics in Brazilian patients: the value of next-generation sequencing for genomic classification. J Investing Med.

9. Hospital MA, Jacquel A, Mazed F, Saland E, Larrue C, et al. (2017) RSK2 is a new Pim2 target with pro-survival functions in FLT3-ITD-positive acute myeloid leukemia. Leukemia.

10. Reiter K, Polzer H, Krupka C, Maiser A, Vick B, et al. (2017) Tyrosine kinase inhibition increases the cells surface localization of FLT3-ITD and enhances FLT3-directed immunotherapy of acute myeloid leukemia. Leukemia.

11. Volpe G, Walton DS, Grainger DE, Ward C, Cauchy P, et al. (2017) Prognostic significance of high GFI1 expression in AML of normal karyotype and its association with a FLT3-ITD signature. Sci Rep 7(1): 11148.

12. Weisberg EL, Puissant A, Stone R, Sattler M, Buhriage SJ, et al. (2017) Characterization of midostaurin as a dual inhibitor of FLT3 and SYK and potentiation of FLT3 inhibition against FLT3-ITD-driven leukemia harbouring activated SYK kinase. Oncotarget 8(32): 52026-52044.

13. Qiu QC, Wang C, Bao XB, Yang J, Shen HJ, et al. (2017) The impact of FLT3 mutations on treatment response and survival in Chinese de novo AML patients. Hematology.

14. Diab S, Abdelaziz AM, Li P, Teo T, Basnet SKC, et al. (2017) Dual inhibition of Mnk2 and FLT3 for potential treatment of acute myeloid leukemia. Eur J Med Chem 139: 762-772.

15. Chen X, Zhang J, Yuan L, Lay Y, Wong YK, et al. (2017) Andrographolide suppresses MVR-11 cell proliferation through the inhibition of FLT3 singling, fatty acid synthesis and cellular iron uptake. Molecules 22(9).

16. Cheng H, Huang C, Xu X, Hu X, Gong S, et al. (2017) PIM1 mRNA expression is a potential prognostic biomarker in acute myeloid leukemia. J Transl Med 15(1): 179.

17. Lu JW, Hsieh MS, Hou HA, Chen CY, Tien HF, et al. (2017) Over expression of SOX4 correlates with poor prognosis of acute myeloid leukemia and is leuwkemogenic in zebra fish. Blood Cancer J 7(8): e593.

18. Si T, Lu Y, Li F, Jiang L, Pei R, et al. (2017) High expression of INHBA is an adverse prognostic factor for de novo acute myeloid leukaemi. Leuk Lymphoma 59(1): 114-120. 


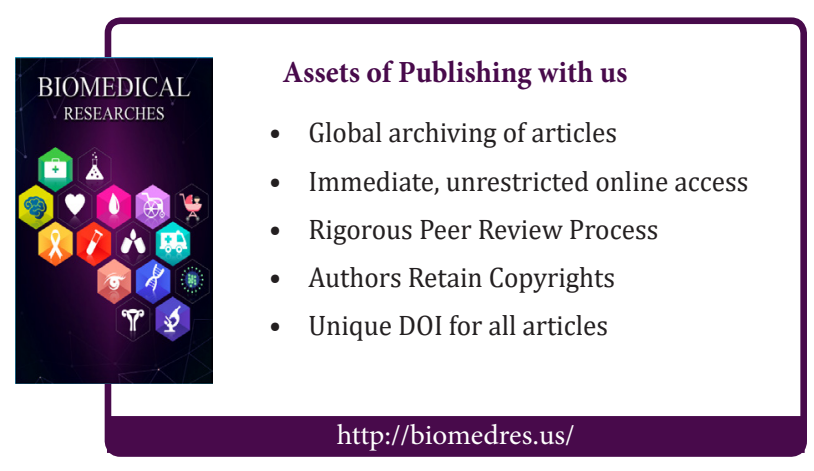

